\title{
Spinal Anesthesia in Infant with Ventriculoperitoneal Shunt: A Case Report of Inguinal Hernia Repair
}

\author{
Gian Matteo Pedrazzi' ${ }^{1}$, Gianfranco Montanari' ${ }^{1}$, Vincenzo Domenichelli2 \\ ${ }^{1}$ Department of Surgery, Unit of Anesthesiology, "Infermi” Hospital, AUSL Romagna, Rimini, Italy \\ ${ }^{2}$ Department of Woman, Newborn, Infants and Child Health, Unit of Pediatric Urology, \\ "Infermi" Hospital, AUSL Romagna, Rimini, Italy \\ Email: zodott@me.com
}

Received 29 March 2016; accepted 14 June 2016; published 17 June 2016

Copyright (C) 2016 by authors and Scientific Research Publishing Inc.

This work is licensed under the Creative Commons Attribution International License (CC BY). http://creativecommons.org/licenses/by/4.0/

c) (i) Open Access

\begin{abstract}
We are describing a case of a female infant with ventriculoperitoneal shunt scheduled for inguinal hernia repair under spinal anesthesia. The child was a premature newborn who, in a recent past, underwent surgery in general anesthesia for retinopathy correction with subsequent difficult mechanical ventilation weaning. The benefit of spinal anesthesia in high-risk infant was described and the risks of spinal anesthesia in the presence of a ventricular shunt device-especially dural leakage and infections were briefly discussed.
\end{abstract}

\section{Keywords}

Spinal Anesthesia, Prematurity, Ventriculoperitoneal Shunt, Inguinal Hernia

\section{Introduction}

Premature infants represent a challenge for pediatric anesthesiologists in fact about six percent of living born infants are delivered prematurely and many of them present clinical complications such as respiratory distress syndrome (RDS), bronchopulmonary dysplasia (BPD), chronic oxygen dependence and chronic airways hyper-reactivity [1]. The structure and function of immature lung predisposes to obstruction, difficulty with ventilation and hypoxia. Apnea of prematurity (AOP) is also a common respiratory pattern in this population, and appears to be related to immaturity of the central respiratory control mechanism, hypothermia, immature musculature and anemia [2]. Apneic episodes are frequent in the preterm infant although they may be precipitated by abrupt changes in pulmonary mechanics, oxygenation, airway stimulation or failure to maintain a patent airway, 
usually occur without a precipitating event (i.e. idiopathic). Anesthetic drugs may further decrease pharyngeal muscle tone and respiratory center output with a difficult return of spontaneous ventilation and complicated mechanical ventilation weaning.

Other two serious complications are retinopathy of prematurity (ROP) and intra-ventricular hemorrhage (IVH); the latter has an incidence of about 25\% - 32\% and often develops subsequent hydrocephalus that needs a shunt device insertion, mainly ventriculoperitoneal shunt (VPS) [3] [4]. Drugs used in general anesthesia often hold neurotoxic properties in this neurologically affected population, thus modern approach for emergency surgery is to limit general anesthesia and improve regional anesthesia whenever possible.

We present a case of a child with VPS scheduled for inguinal hernia repair who undergoes low abdominal surgery under spinal anesthesia (SA) without complications.

\section{Case Description}

The patient was a female born at 24th weeks gestational age (GA) after spontaneous vaginal delivery. Birth weight (BW) was $0.640 \mathrm{~kg}$. She was immediately mechanically ventilated in neonatal intensive care unit (NICU) which was continued for fifteen days because of RDS, anemia and tetraventricular hemorrhage with subsequent obstructive hydrocephalus.

At 28th weeks gestational age she underwent VPS insertion. At 46th weeks GA the newborn developed severe ROP and therefore underwent urgent laser therapy and vitrectomy under general anesthesia with post-operative respiratory distress followed by sixteen days of mechanical ventilation with difficult ventilatory weaning. After three months the baby had hospital admittance because of an incarcerated right inguinal hernia with abdominal pain.

Pediatric surgeons decided for a urgent surgical procedure and after a fast pre-operative screening (blood count, coagulation tests and electrocardiogram) the neonate was moved to the operating theatre.

The anesthesists decided to perform a spinal anesthesia to preserve spontaneous breathing and avoid mechanical ventilation.

The baby girl weighted $5.1 \mathrm{~kg}$ and hematocrit was 35\% with a normally functioning VPS (neurosurgical consultation was performed). The baby received antibiotic prophylaxis (50 $\mathrm{mg} / \mathrm{kg}$ of ceftriaxone i.v.), and SA was performed according to our institutions guidelines [5] [6]. Before the surgical procedure the operating room was prepared to improve patient comfort with air temperature above 22 Celsius degrees, ambient dim lights, and soft classical music to promote natural sleeping.

She received a $0.8 \mathrm{mg} / \mathrm{kg}$ spinal $0.5 \%$ hyperbaric bupivacaine in lateral decubitus and she was monitored with intermittent non invasive blood pressure (left leg cuff position), three leads electrocardiography and continuous oxygen blood saturation [7] [8]. SA was performed at L4-L5 lumbar level of puncture with a 25 gauge- $25 \mathrm{~mm}$ length pencil point needle. A little amount of lidocaine anesthetic cream (about $3 \mathrm{~cm} 2$ surface) was applied to the back skin 30 minutes before the puncture. The surgical procedure lasted 43 minutes with a good anesthesia quality (T5 sensory block level) and without intra-venous sedation/analgesia request. Post-operative pain management started with $20 \mathrm{mg} / \mathrm{Kg}$ rectal acetaminophen.

The baby presented normal cardiovascular parameters (no systolic pressure below $83 \mathrm{mmHg}$ ) with normal respiratory rate. No oxygen support was necessary with no arterial oxygen desaturation during medical procedure. The post-anesthesia recovery was performed in post-anesthesia care unit without complications with a fast and full sensory/motor recovery after 65 minutes from SA and with a continuous urine output above 1.5 $\mathrm{ml} / \mathrm{Kg} / \mathrm{h}$.

No central temperature above 37.3 Celsius degrees was recorded.

The baby was discharged home 48 hours later without complications.

\section{Discussion}

VPS placement is the mainstay in the treatment of infants hydrocephalus (congenital or acquired) and the main indication in premature population is non-responding IVH to medical treatment with obstructive hydrocephalus [1].

Premature infants may require various surgical procedures and nearly $30 \%$ have inguinal hernia, which are bilateral in about $20 \%$ of patients [9].

Inguinal hernia is more frequent in premature infant with VPS (47\%), probably because the amount of drained 
cerebrospinal fluid (CSF) in abdomen increases intra-abdominal pressure [10]. One of the most important VPS complication is VPS infection, with an infection rate as high as $38 \%$ and four-fold increase rate in premature neonates [11] [12]. The most common pathogens involved are Staphylococcus epidermidis, Staphylococcus aureus and GRAM negative bacteria [12] [13]. Although no controlled studies show a significant decreasing infection rate with antibiotic prophylaxis, meta-analysis and experts opinion suggest perioperative antibacterial prophylaxis, particularly targeted on Staphylococcus spp. [14]. Many regimens are described including vancomycin, rifampicin, nafcillin and cephalosporins as in our institution [15].

Abdominal surgery in premature infants with VPS is usually performed under general anesthesia, Although defined contraindications to SA are not mentioned, many anesthesiologists refrain from using SA in this patients because of the fear of VPS infections.

In literature, we can find the same incident of meningitis after SA as after general anesthesia.

In adult population, the overall incident of bacterial infection after lumbar puncture is estimated $0.002 \%$ [16]). In children, the exact incidence of meningitis after SA is unknown, and the large study of Giaufre et al. (24.409 patients) reveals that only $2 \%$ of all regional anesthesias performed in children are spinal anesthesias and that only 30 patients are prematures [17]. Luz et al. report a case of fatal bacterial meningitis after SA in a preterm infant, but cannot exactly determine if the infection is consequent or just coincident with the lumbar puncture [18]. Easley et al. describe a case of aseptic meningitis after SA in a 2.5 month former premature but no causal relationship with SA is possibly proved [19].

Concerns that dural leakage following SA in children with VPS may compromise CSF flow and shunt performance seem to be overestimated [20]. During SA, we note a less CSF flow through the pencil point needle (about one drop of CSF/2.5 seconds versus normally one drop of CSF/1 second) probably due to a different CSF shunted flow into the spinal chord.

Preterm infants appear to represent a very high risk population for anesthesia in fact, most of them present chronic lung dysfunctions and the risk of a difficult ventilatory weaning after general anesthesia is very high.

In our institution, we decide to use SA as the first choice anesthesia to manage preterm newborns trying to limit perioperative respiratory complications avoiding general anesthesia.

In conclusion, we think that future studies comparing respiratory, surgical and neurological outcomes in preterm patients with VPS in general vs spinal anesthesia are indicated. Antibacterial prophylaxis is recommended and a strict aseptic technique is mandatory.

SA in experienced hands may be considered as a safe alternative to general anesthesia in selected patients. A further mandatory effort to improve children's safety is to build a very harmonious working team, in fact a not perfect integration of surgeons, anaesthesiologists and nurses staff may compromise the outcomes of those little patients.

\section{References}

[1] Gregory, G.A. (2002) Anesthesia for Premature Infants. In: Gregory, G.A., Ed., Pediatric Anesthesia, 4th Edition, Churchill Livingstone, New York, 345-348, 369-376, 400.

[2] Henderson-Smart, D.J. (1995) Recurrent Apnea. Bailliere’s Clinical Pediatric, 3, 203-222.

[3] Van de Bor, M., Verloove-Vanhorick, S.P., Brand, R., et al. (1987) Incidence and Prediction of Periventricular-Intraventricular Hemorrage in Very Preterm Infants. Journal of Perinatal Medicine; 15, 333-339. http://dx.doi.org/10.1515/jpme.1987.15.4.333

[4] Wildrick, D. (1997) Intraventricular Hemorrage and Long-Term Outcome in the Premature Infant. Journal of Neuroscience Nursing, 29, 281-289. http://dx.doi.org/10.1097/01376517-199710000-00002

[5] Langley, J.M., Le Blanc, J.C., Drake, J., et al. (1993) Efficacy of Antimicrobial Prophylaxis in Placement of Cerebrospinal Fluid Shunts: Meta-Analysis. Clinical Infectious Diseases, 17, 98-103. http://dx.doi.org/10.1093/clinids/17.1.98

[6] Rotim, K., Miklic, P., Paladino, J., et al. (1997) Reducing the Incidence of Infection in Pediatric Cerebrospinal Shunt Operations. Child's Nervous System, 13, 584-587. http://dx.doi.org/10.1007/s003810050144

[7] Williams, J.M., Stoddart, P.A., Williams, S.A.R., et al. (2001) Postoperative Recovery after Inguinal Herniotomy in Ex-Premature Infants: Comparison between Sevoflurane and Spinal Anesthesia. British Journal of Anaesthesia, 86, 366-371. http://dx.doi.org/10.1093/bja/86.3.366

[8] Puncuh, F., Lampugnani, E. and Kokki, H. (2005) Spinal Anaesthesia in Pediatric Patients. Current Opinion in Anesthesiology, 18, 299-305. http://dx.doi.org/10.1097/01.aco.0000169239.36927.8b 
[9] Harper, R.G., Garcia, A. and Sia, C. (1975) Inguinal Hernia; a Common Problem of Premature Infants Weighing 1000 Grams or Less at Birth. Pediatrics, 56, 112-115.

[10] Moazam, F., Glenn, J.D., Kaplan, B.J., et al. (1984) Inguinal Hernia after Ventriculoperitoneal Shunt Procedures in Pediatric Patients. Journal of the American College of Surgeons, 159, 570-572.

[11] McGirth, M.J., Zaas, A., Fuchs, H.E., et al. (2003) Risk Factor for Pediatric Ventriculoperitoneal Shunt Infection and Predictors of Infectious Pathogens. Clinical Infectious Diseases, 36, 858-862. http://dx.doi.org/10.1086/368191

[12] Bruisma, N., Stobberingh, E.E., Herpers, M.J., et al. (2000) Subcutaneous Ventricular Catheter Reservoire and Ventriculoperitoneal Drain-Related Infections in Preterm Infants and Young Children. Clinical Microbiology and Infection, 6, 202-206. http://dx.doi.org/10.1046/j.1469-0691.2000.00052.x

[13] Davis, S.E., Levy, M.L., Gordon McComb, J., et al. (1999) Does Age or Other Factors Influence the Incidence of Ventriculoperitoneal Shunt Infections? Pediatric Neurosurgery, 30, 253-257. http://dx.doi.org/10.1159/000028806

[14] Haines, S.J. and Walters, B.C. (1994) Antibiotic Prophylaxis for Cerebrospinal Fluid Shunts: A Meta Analysis. Neurosurgery, 34, 87-92. http://dx.doi.org/10.1227/00006123-199401000-00012

[15] Arnaboldi, L. (1996) Antimicrobial Prophylaxis with Ceftriaxone in Neurosurgical Procedures. A Prospective Study of 100 Patients Undergoing Shunt Operations.Chemotherapy, 42, 384-390. http://dx.doi.org/10.1159/000239470

[16] Eng, R.H.K. and Seligman, S.J. (1981) Lumbar Puncture Induced Meningitis. JAMA, 245, 1456-1459. http://dx.doi.org/10.1001/jama.1981.03310390056023

[17] Giaufre, E., Dalens, B. and Gombert, A. (1996) Epidemiology and Morbidity of Regional Anesthesia in Children: A One-Year Prospective Survey of the French-Language Society of Pediatric Anesthesiologists. Anesthesia \& Analgesia, 83, 904-912.

[18] Luz, G., Buchele, H., Innerhofer, P., et al. (1999) Spinal Anesthesia and Meningitis in Former Preterm Infants: CauseEffect? Pediatric Anesthesia, 9, 262-264.

[19] Easley, R.B., George, R., Connors, D., et al. (1999) Aseptic Meningitis after Spinal Anesthesia in an Infant. Anesthesiology, 91, 305-307. http://dx.doi.org/10.1097/00000542-199907000-00040

[20] Ludmyla, K., Platis, C.M., et al. (2006) Spinal Anesthesia in Infants with Ventriculoperitoneal Shunt: Report of Five Cases and Review of Literature. Pediatric Anesthesia, 16, 578-583. http://dx.doi.org/10.1111/j.1460-9592.2005.01794.x 